\title{
Advanced Decentralized DER Control for Islanded Microgrids
}

\author{
M. J. Hossain and J. Lu \\ Queensland Micro and Nanotechnology Centre \\ Griffith School of Engineering \\ Griffith University, Gold Coast, Australia \\ j.hossain@griffith.edu.au, j.lu@griffith.edu.au
}

\author{
M. A. Mahmud ${ }^{1}$ and T. Aziz ${ }^{2}$ \\ ${ }^{1}$ School of Software and Electrical Engineering, \\ Swinburne University of Technology, Melbourne, Australia. \\ ${ }^{2}$ Faculty of Engineering, American International University- \\ Bangladesh, Dhaka, Bangladesh.
}

\begin{abstract}
Voltage and frequency regulations are main requirements for autonomous operation of an isolated microgrid with electronically interfaced distributed energy resource (DER) units. This paper presents a robust decentralized control design scheme for a multi-DER microgrid to enhance its voltage and frequency regulations. The proposed control scheme utilizes linear quadratic (LQ) decentralized control scheme to design controllers, which are robust to the uncertainty in the network and system nonlinearities. The effectiveness of the proposed controller is demonstrated through simulation on a test system, with complete nonlinear models, for large disturbances. It is found that the designed controller provides excellent performance during transient periods as well as restores the pre-disturbance steady-state operation within prescribed time frame.
\end{abstract}

Index Terms-- DER units, microgrid, distribution systems, linear quadratic control, robust control.

\section{INTRODUCTION}

During the next decade, microgrids will emerge as a major enabler of the smart grid for the integration of small and medium sized DER units into the electricity grid. Microgrids offer a promising cost effective solution to the integration of renewable energy with reduced losses, lower transmission and distribution costs, higher energy efficiency, and a number of environmental and economic benefits [1]. However, the coexistence of multiple energy resources with versatile dynamic properties and electrical characteristics has raised concerns over the stability, control and efficiency of microgrids. The control and operation of microgrids are challenging especially during islanded mode due to the none/less inertial, dispatchable and non-dispatchable and nonfirm characteristics of DER units. These characteristics of DER units might give rise to load-tracking problems in microgrid islanded mode of operation.

In order to ensure stability in transients as well as steady states, DER units in autonomous mode use local control and maintain the voltage and frequency within their predefined limits. The real and reactive powers are controlled so that the DER units properly share the total load by regulating frequency and voltage. This control must be reliable, fast and robust because when voltage magnitude and frequency undergo unacceptable excursions, the microgrid collapses completely. Although centralized DER control is theoretically possible, decentralized scheme based on droop characteristics offers cost effective and efficient solution. It uses only local measured signals and does not require large data transfer and reliable communication links [2].

Microgrid control is a complex issue, and is consequently the subject of increasing research efforts around the world. The control scheme proposed in [3] contains inner voltage control loop for controlling the three-phase inverter voltage. It proposes an external power control loop for controlling real and reactive power flow and hence, facilitates power sharing among DER units when fault occurs and microgrid islands. A voltage power droop/frequency reactive boost control scheme is proposed in [4] that allows multiple voltage source converters (VSCs) to operate in parallel. Reference [5] proposes a modified droop in order to force the non-dispatchable generator to share the load according to their available input mechanical powers. The authors in [6] propose a microgrid voltage and frequency control scheme which is synthesized to allow converters with standard inductor interface and $d q$ (direct and quadrature axis)-frame current control to operate in intentional islanding mode. The developed model and control strategies in [3-6] are linear and based on pre-specified circuit configurations, which may not necessarily guarantee stability and robustness under large disturbances or a change in network configuration.

Robust control techniques are proposed in [7-11] to address the problem of linear controllers. In [7] linear quadratic Gaussian method is used to design a robust controller considering known load configuration and uncertain parameters. Reference [8] presents a control structure for microgrid converters based on direct-voltage control and optimized dynamic power sharing. Model uncertainty and load disturbances are considered in designing $\mathrm{H}_{\infty}$ control for voltage and current loop in [9]. However, in [7-11] nonlinearities of the switching converters are not considered. The uncertainty in DER units is neglected in [12]. A centralized control technique is used in [14] which is not practical for enormous DER units in the distribution systems. 
During the switching of microgrids from grid connected to islanded mode, the operating point move far away from the nominal operating point. This paper presents a robust control technique to design controllers for converters considering nonlinearities and operating point deviations. The designed controller provides guaranteed performance and stability characteristics over a pre-specified region around the equilibrium point.

The rest of the paper is organized as follows: Section II provides the mathematical modeling of a microgrid system with DER units. The proposed control strategy and uncertainty modeling are presented in Section III and the control design algorithm is described in Section IV. The performance of the controller is demonstrated through a series of nonlinear simulations in Section V. Concluding remarks and suggestions for future work are given in Section VI.

\section{MODELING OF THE MICROGRID}

A schematic diagram of a typical microgrid is shown in Fig. 1 where $V_{d c i}$ is an ideal DC voltage source equivalent to a DER. Fig. 2 shows the single line diagram of two subsystems. where $R_{f i}$ is the equivalent resistance accounting for the switching and transformer losses, $L_{f i}$ is the filter and leakage reactance of the transformer, $V_{L i}$ is the voltage across the RLC load, and $R_{t}$ and $L_{t}$ are line resistance and inductance, respectively.

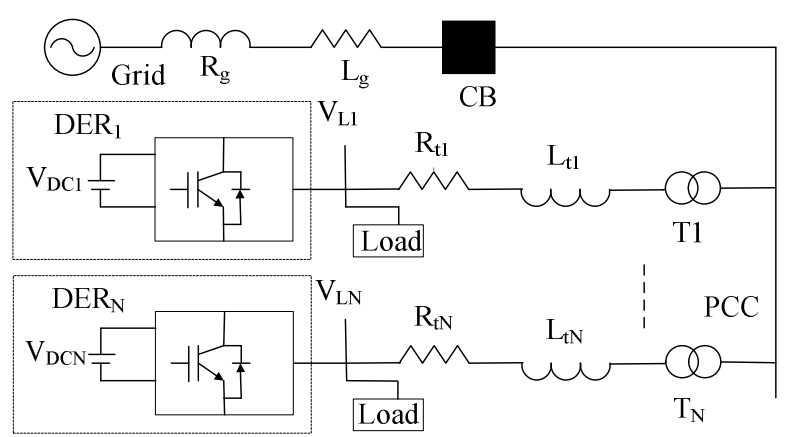

Fig. 1. Schematic diagram of a microgrid subsystem.

Subsystem j

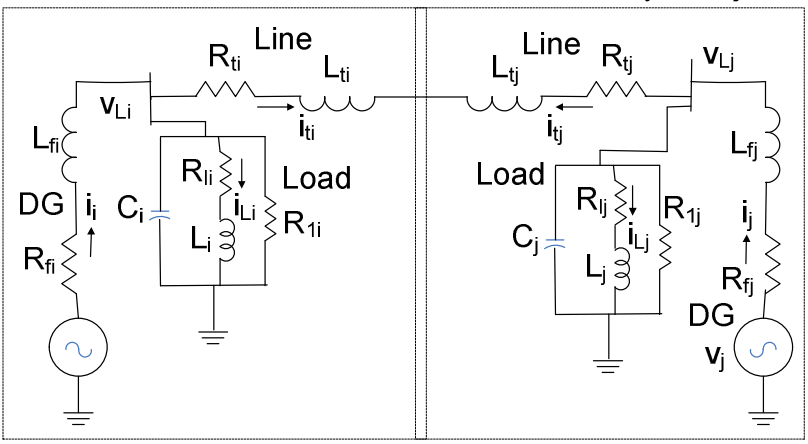

Fig 2. Single line diagram of microgrid subsystem.

The mathematical modeling of $i t h$ subsystem in $d q$ frame is given by equations (i)-(viii) [11]:
$L_{f i} \dot{I}_{d i}=V_{d i}-R_{f i} I_{q i}+\omega_{i} I_{q i}-V_{L d i}$

$L_{f i} \dot{I}_{q i}=V_{q i}-R_{f i} I_{d i}-\omega_{i} I_{d i}-V_{L q i}$

$L_{t i} \dot{I}_{t d i}=V_{L d i}-R_{t i} I_{q i}+\omega_{i} I_{t q i}-V_{L d j}$

$L_{t i} \dot{I}_{t q i}=V_{L d i}-R_{t i} I_{d i}-\omega_{i} I_{t d i}-V_{L q j}$

$C_{i} \dot{V}_{L d i}=I_{d i}-I_{t d i}+\omega_{i} V_{L q i} C_{i}-I_{c d i}-1 / R_{l i} V_{L q i}$

$C_{i} \dot{V}_{L q i}=I_{q i}-I_{t q i}-\omega_{i} V_{t d i} C_{i}-I_{c q i}-1 / R_{l i} V_{L d i}$

$L_{i} \dot{I}_{L d i}=V_{L d i}-R_{l i} I_{L q i}+\omega_{i} I_{L q i} L_{i}$

$L_{i} \dot{I}_{L q i}=V_{L q i}-R_{l i} I_{L d i}-\omega_{i} I_{L d i} L_{i}$

where $V_{i}=\sqrt{V_{d i}^{2}+V_{q i}^{2}}$. The real and reactive power injected by the DER at the $i t h$ bus is given by:

$$
\begin{aligned}
& P_{i}=\sum_{j=1}^{N}\left|V_{L i}\right|\left|V_{L j}\right|\left(G_{i j} \cos \delta_{i j}+B_{i j} \sin \delta_{i j}\right) \\
& Q_{i}=\sum_{j=1}^{N}\left|V_{L i}\right|\left|V_{L j}\right|\left(G_{i j} \sin \delta_{i j}+B_{i j} \cos \delta_{i j}\right)
\end{aligned}
$$

The stator vector for subsystem $i$ is

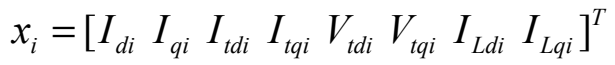

The measured output and control input are

$y_{i}=\left[V_{t d i} V_{t q i}\right]^{T}, u_{i}=\left[V_{d i} V_{q i}\right]^{T}$. The reference voltage and voltage source angle are obtained using the following droop control law:

$\dot{\delta}_{i}^{*}=-m_{i}\left(P_{i}-P_{\text {ratedi }}\right)$

$V_{i}^{*}=V_{\text {ratedi }}-n_{i}\left(Q_{i}-Q_{\text {rated }}\right)$

where superscript * represents reference value, $\mathrm{m}$ and $\mathrm{n}$ are droop coefficients and values of droop coefficients are taken from [12].

\section{CONTROL STRATEGY AND UNCERTAINTY}

Fig. 3 illustrates a schematic diagram of the DER control system in which host bus voltage and frequency are regulated.

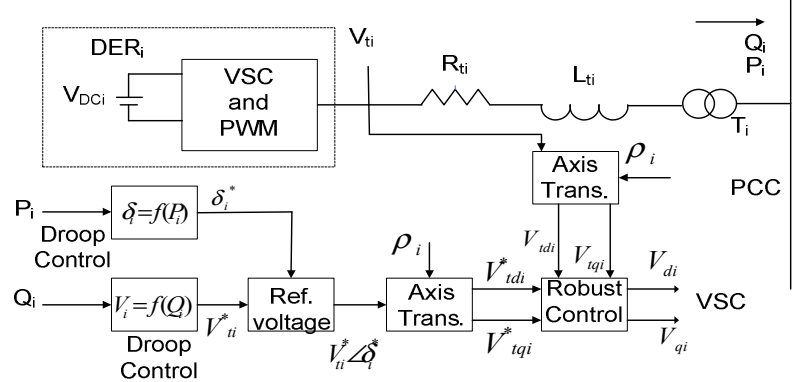

Fig. 3. Block diagram of DER control strategy.

The real and reactive power output of the DER are used to calculate $\delta_{i}^{*}$ and $V_{i}^{*}$ using the droop control strategy in equation (x)and $p_{i}$ is the $d q$-frame reference angle. The robust decentralized control scheme is shown in Fig. 4 in which local uncertainty includes system nonlinearities and the other 
uncertainty due to interconnection of DER units. One controller is designed for each DER unit.

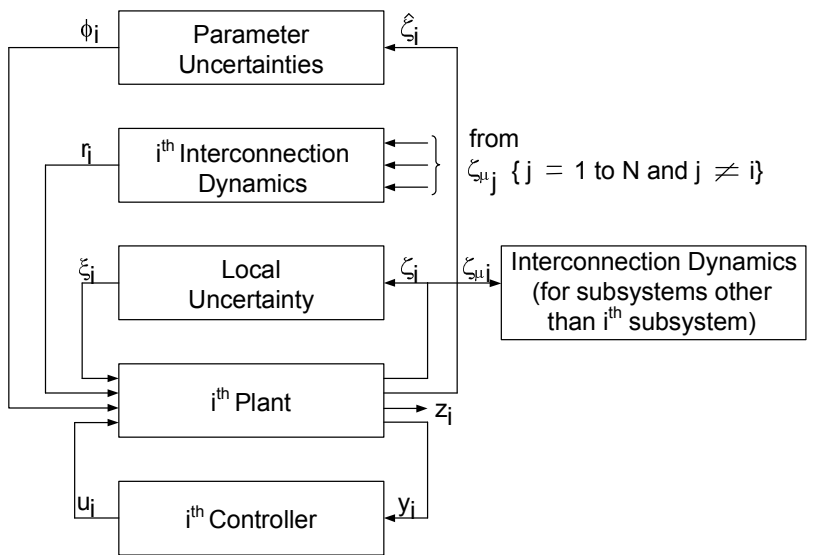

Fig. 4. Block diagram of robust decentralized control.

The power system model (i)-(viii) is described by the following large-scale system comprising $N$ number of subsystems denoted by $S_{i}, i=1,2, \ldots . N[13]$ :

$S_{i}: \dot{x}(t)=A_{i} x_{x}(t)+B_{i} u_{i}(t)++E_{i} \xi_{i}(t)+L_{i} r(t)$

$z_{i}(t)=C_{i} x_{x}(t)+D_{i} \xi_{i}(t)$

$\zeta_{i}(t)=G_{i} x_{x}(t)+H_{i} u_{i}(t)$

$y_{i}(t)=C_{y i} x_{x}(t)+D_{y i} \xi_{i}(t)$

where $x_{i} \in R^{n i}$ is the state vector, $u_{i} \in R^{m i}$ the control input, $\xi_{i} \in R^{p i}$ the perturbation, $\zeta_{i} \in R^{h i}$ the uncertainty output, $z_{i} \in R^{q i} \quad$ the controlled output, $y_{i} \in R^{g i}$ the measured output, and the input $r_{i}$ describes the effect of the other subsystems $\left(S_{1}, \ldots, S_{i-1}, S_{i+1}, \ldots, S_{N}\right)$ on subsystem $S_{i}$. The complete dynamic system is linearized about the desired equilibrium point: one part consists of the states of the devices in the subsystem $\left(x_{i}\right)$ and the other with the rest of the states $\left(r_{i}\right)$; the matrices $A_{i}, B_{i}$ and $C_{y i}$ are appropriately chosen from the complete linearized model equations. In this paper measured outputs $\left(y_{i}\right)$ and control output $\left(z_{i}\right)$ are similar signals, i.e. $y_{i}=z_{i}=\left[V_{t d i} V_{t q i}\right]^{T}$ and $u_{i}=\left[V_{d i} V_{q i}\right]^{T}$.

The most challenging problem is the determination of uncertainty matrices $E_{i}$ and $L_{i}$ which includes the uncertainty due to system nonlinearity and interconnection. A new reformulation technique proposed in [14] is used to quantify the higher order terms which are generally neglected in Taylor series for conventional linearization. The nonlinear converters of DER units are modelled as a linear part plus a nonlinear part, the nonlinear term being the Cauchy remainder term in the Taylor series expansion. Bounds derived for this Cauchy remainder term are used to define an uncertain linear model for which a robust control design is performed. In this paper, in the design of the linear controller, the Cauchy remainder is incorporated as an uncertain term thus quantifying the deviations from the equilibrium point. The procedure for the new reformulation is described in [14].
This reformulation is used to design the robust controller for DER units. The robust controller as shown in Fig. 3 is designed using the equations [13]:

$$
\begin{aligned}
& \dot{x}_{c i}=\left\{A_{i}-\left[B_{i} R_{i}^{-1} B_{i}^{T}-\bar{B}_{2 i} \bar{B}_{2 i}^{T}\right] X_{i}\right\} x_{c i} \\
& +\left[Y_{i}-X_{i}\right]^{-1} C_{y i}^{T} W_{i}^{-1}\left[y_{i}(t)-C_{y i} x_{c i}\right] \\
& u_{i}=-R_{i}^{-1} B_{i}^{T} X_{i} x_{c i} .
\end{aligned}
$$

where $X_{i}$ and $Y_{i}$ are the the solutions to the following pair of parameter-dependent coupled algebraic equations [13]:

$$
\begin{array}{r}
A_{i}^{T} Y_{i}+Y_{i} A_{i}+Y_{i} \bar{B}_{2 i} \bar{B}_{2 i}^{T} Y_{i}-\left[C_{y i}^{T} W_{i}^{-1} C_{y i}-\bar{C}_{i}^{T} \bar{C}_{i}\right]=0, \\
\text { (xvii) } \\
A_{i}^{T} X_{i}+X_{i} A_{i}+\bar{C}_{i}^{T} \bar{C}_{i}-X_{i}\left[B_{i} R_{i}^{-1} B_{i}^{T}-\bar{B}_{2 i} \bar{B}_{2 i}^{T}\right] X_{i}=0
\end{array}
$$

where $R_{i}=\bar{D}_{i}^{T} \bar{D}_{i}, W_{i}=\bar{D}_{y i} \bar{D}_{y i}^{T}$ and $\bar{\theta}_{i}=\sum_{n=1, n \neq i}^{N} \theta_{n}$,

$$
\begin{aligned}
& \bar{C}_{i}=\left\lfloor\begin{array}{l}
C_{i} \\
\left(\tau_{i}+\bar{\theta}_{i}\right)^{1 / 2} H_{i}
\end{array}\right\rfloor, \bar{D}_{i}=\left\lfloor\begin{array}{l}
D_{i} \\
\left(\tau_{i}+\bar{\theta}_{i}\right)^{1 / 2} G_{i}
\end{array}\right\rfloor, \\
& \bar{B}_{2 i}=\left\lfloor\begin{array}{lll}
\tau_{i}^{-1 / 2} E_{i} & \theta_{i}^{-1 / 2} L_{i}
\end{array}\right\rfloor, D_{y i}=\left\lfloor\begin{array}{lll}
\tau_{i}^{-1 / 2} D_{y i} & 0
\end{array}\right\rfloor .
\end{aligned}
$$

For complete description of the control design scheme, please see [13].

\section{IV. CONTROL ALGORITHM}

The test system as shown in Fig. 5 (included after the reference section) is divided into two subsystems and a multivariable controller is designed for each subsystem. The control design algorithm is implemented using the following steps:

(i) Model the microgrid using equations (i)-(viii) and solve it for the steady state.

(ii) Reformulate the nonlinear system using the technique proposed in [14] and determine the matrices given in (xi)-(xiv). From the nonlinear simulations of the faulted system including intentional islanding, obtain the range of the variation of all the state variables.

Quantify the uncertain term from the analytical expression developed in [14] and check that it satisfies the norm bound constraint [13].

(v) Solve the Ricatti equations (xvii) and (xviii).

(vi) The controller is given by equations (xv) and (xvi).

\section{CASE STUDIES}

The single line diagram of the test microgrid is shown in Fig. 5. The parameters for this microgrid are given in [15]. The system consists of three $13.8 \mathrm{kV}$ feeders which are connected to the large system through a $69 \mathrm{kV}$ radial line. The total load of the system is 7.3 MW and 3.97 MVAR. Out of five loads, four loads are linear and the other one is a nonlinear diode rectifier load. There are two DER units in the system, a photovoltaic generator and a full converter based 
wind turbine. Both generators are connected to the system via voltage source converters (VSC). Each DER unit is modeled with a constant DC voltage source, an insulated gate bipolar transistor based VSC and a filter. Each distribution line is represented by lumped series RL branches. The performance of the controller is tested for the following disturbances to verify the robustness performance:

\section{(A) Severe Three Phase Fault}

The objective of this case study is to demonstrate that the designed controller can ensure stability by maintaining frequency and voltage within statutory limits and share load among DERs during a severe three phase fault in grid connected mode. A three phase fault is applied at $1 \mathrm{~s}$ on PCC and cleared after five cycles. Figs. 6 and 7 show the terminal voltage and reactive power output of $\mathrm{DER}_{1}$. It is clear from the figures that the proposed controller ensures stable operation during severe transients and the post fault condition. Each DER controller autonomously and effectively responds to the change in system configuration. From the simulated results it can be seen that the system returns to steady state within $0.1 \mathrm{~s}$ with zero steady state error.

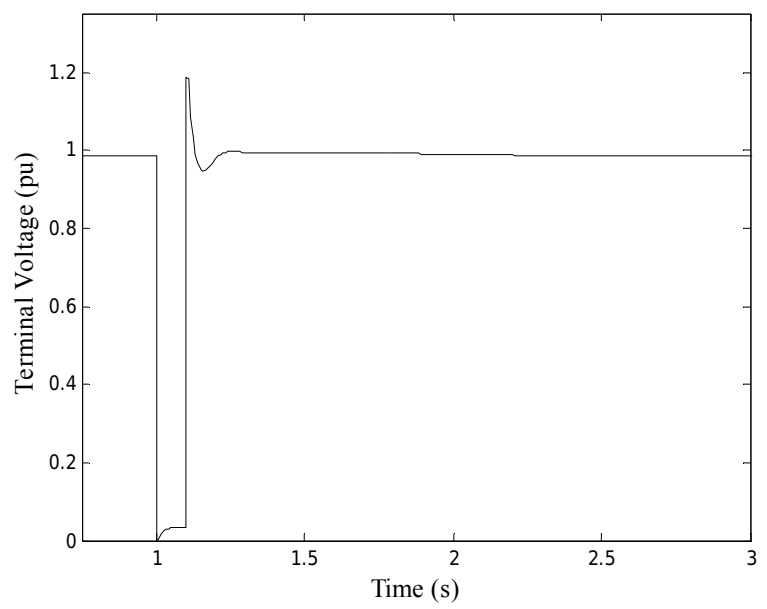

Fig. 6. Terminal voltage of $\mathrm{DER}_{2}$ for three phase fault on PCC.

\section{(B) Intentional Islanding}

In this case the performance of the controller is investigated for pre-planned islanding. Initially the microgrid was working in a grid connected mode. Before the islanding $\mathrm{DER}_{1}$ and $\mathrm{DER}_{2}$ were supplying $3 \mathrm{MW}$ and $3.5 \mathrm{MW}$, respectively and the remaining $1 \mathrm{MW}$ was imported from the grid. At $\mathrm{t}=1 \mathrm{~s}$, an intentional islanded command is applied to the $69 \mathrm{kV}$ line breakers and the real power set point of $\mathrm{DER}_{2}$ is changed from 3.5 MW to $4.5 \mathrm{MW}$ through the action of its governor. Figs. 8 and 9 show the real power sharing between DER units and terminal voltage excursions. Both the DER units control their reactive power to control the terminal voltage. From this study it is clear that the proposed controller can ensure stability during switching from grid connected to islanded mode and provides robust performance.

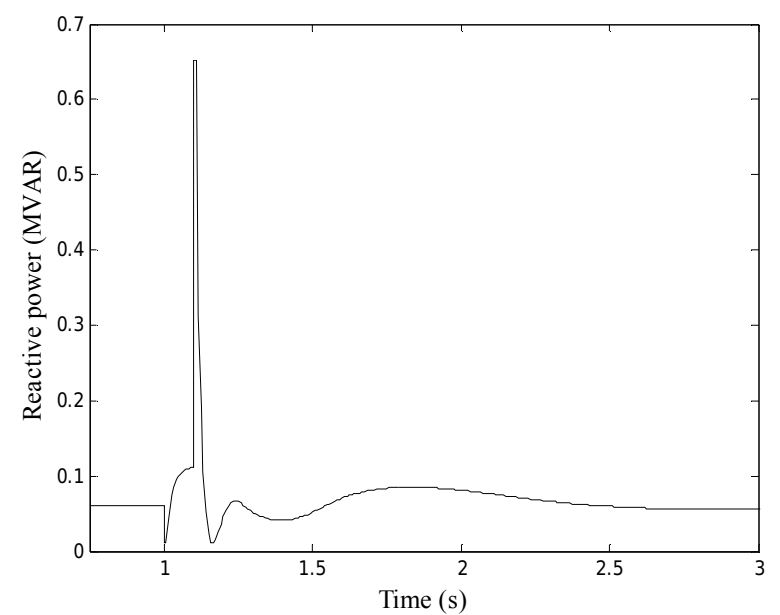

Fig. 7. Reactive power of $\mathrm{DER}_{2}$ for three phase fault on PCC.

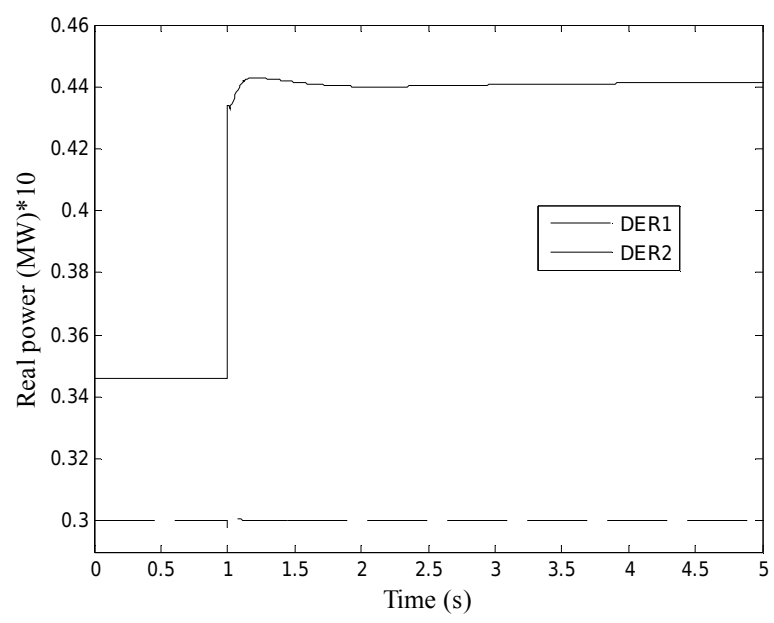

Fig. 8. Real power output of DER during islanding.

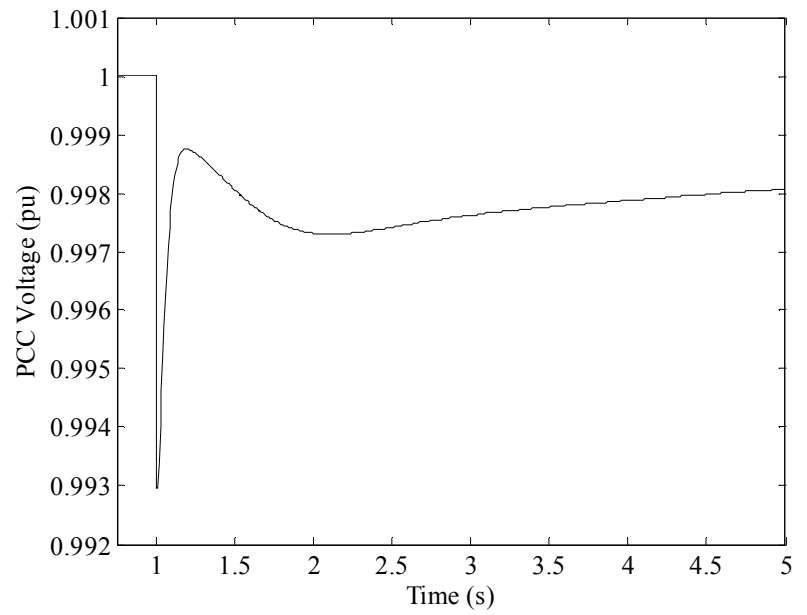

Fig. 8. PCC voltage during islanding. 


\section{CONCLUSIONS}

In this paper a systematic procedure for designing robust decentralized DER controllers is presented, which eventually enhances the voltage and frequency stability of islanded microgrids. The controller is designed based on local measurement signals and the appropriate inclusion of uncertainty in the design process enhances the robustness of the controller. The designed controller guarantees stability if the system post-fault operating point falls in the region for which the controller is designed. It provides feasible and smooth transition of the microgrid from the grid connected to islanded mode operation. The simulation studies validate the desired performance of the microgrid subject to severe three phase fault and intentional islanding and hence, prove the efficacy and robustness of the designed controller. The future aim of this work is to include the dynamics of sensitive loads in the design process of the controller.

\section{REFERENCES}

[1] N. Hatziargyriuo, H. Assano, R. Iravani and C. Marnay, "Microgrids," IEEE Power and Energy Magazine, vol. 5, no. 4, pp. 78-94, JulyAugust 2007.

[2] J. A. P. Lopes, C. L. Moreira and A. G. Madureira, "Defining control strategies for microgrid islanded operation," IEEE Trans. on Power Systems, vol. 21, no. 2, pp. 916-923, May 2006.

[3] Y. W. Li, D. M. Vilathgamuwa and P. C. Loh, "Design, analysis, and real-time testing of a controller for multibus microgrid system," IEEE Transactions on Power Electronics, vol. 19, no.5, pp. 1195- 1204, Sept. 2004

[4] C. K. Sao and P. E. Lehn, "Control and Power Management of Converter Fed Microgrids," IEEE Transactions on Power Systems, vol. 23, no. 3, pp. 1088-1098, Aug. 2008.

[5] M. Fazeli, G. M. Asher, C. Klumpner, L. Yao and M. Bazargan, "Novel
[6] C. K. Sao and P. W. Lehn, "Intentional islanded operation of converter fed microgrids," IEEE Trans. On Power delivery, vol. 20, no. 2, pp. 1009-1016, April 2005.

[7] H. Karimi, H. Nikkhajoei and R. Iravani, "A linear quadratic Gaussian controller for a stand-alone distributed resource unit-simulation case studies," in Proc. 2007 IEEE Power Engineering Society General Meeting Conf., pp. 1-6.

[8] M. B. Delghabi, "Advanced islanded-mode controller of microgrids," Ph.D. dissertation, Dept. Elec. and Com. Eng., Univ. Western Ontario, Ontario, Canada, 2011.

[9] Y. A.-R. I. Mohamed, H. H. Zeineldin, M. M. A. Salama and R. R. Seethapathy, "Seamless Formation and Robust Control of Distributed Generation Microgrids via Direct Voltage Control and Optimized Dynamic Power Sharing," IEEE Transactions on Power Electronics, vol. 27, no. 3, pp. 1283-1294, March 2012.

[10] Chun-xia Dou, Fang Zhao, Zhi-qian Bo, Xing-bei Jia and Dong-le Liu, "Hळ robust control of DC-AC interfaced microsource in microgrids," in Proc. 2011 IEEE Power Engineering and Automation Conf., pp.478-485, 8-9 Sept. 2011.

[11] A. H. Etemadi, E. J. Davison and R. Iravani,"A Decentralized Robust Control Strategy for Multi-DER Microgrids-Part I: Fundamental Concepts," IEEE Transactions on Power Delivery, vol. 27, no. 4, pp. 1843-1853, Oct. 2012.

[12] R. Majumder, A. Ghosh, G. Ledwich and F. Zare, "Power Management and power flow control with back-to-back converters in a utility connected microgrid," IEEE Transactions on Power Systems, vol. 25, no. 2, pp. 821-834, May 2010.

[13] M. J. Hossain, H. R. Pota and C. Kumble, "Decentralized robust static synchronous compensator control to augment dynamic transfer capability,", Journal of Renewable and Sustainable Energy, vol. 2, no. 2, pp. 022701(1)-022701(20), March 2010.

[14] M. J. Hossain, H. R. Pota, V. Ugrinovskii, and R. A. Ramos, "Simultaneous STATCOM and pitch angle control for improved fault ride-through capability of fixed-speed wind turbines," IEEE Trans. on Sustainable Energy, vol. 1, no. 3, pp. 142-151, October 2010.

[15] F. Katiraei, M. R. Iravani and L. W. Lehn, "Microgrid autonomous operation during and subsequent to islanding process," IEEE Transaction on Power Delivery, vol. 20, no. 1, pp. 248-257, January 2007

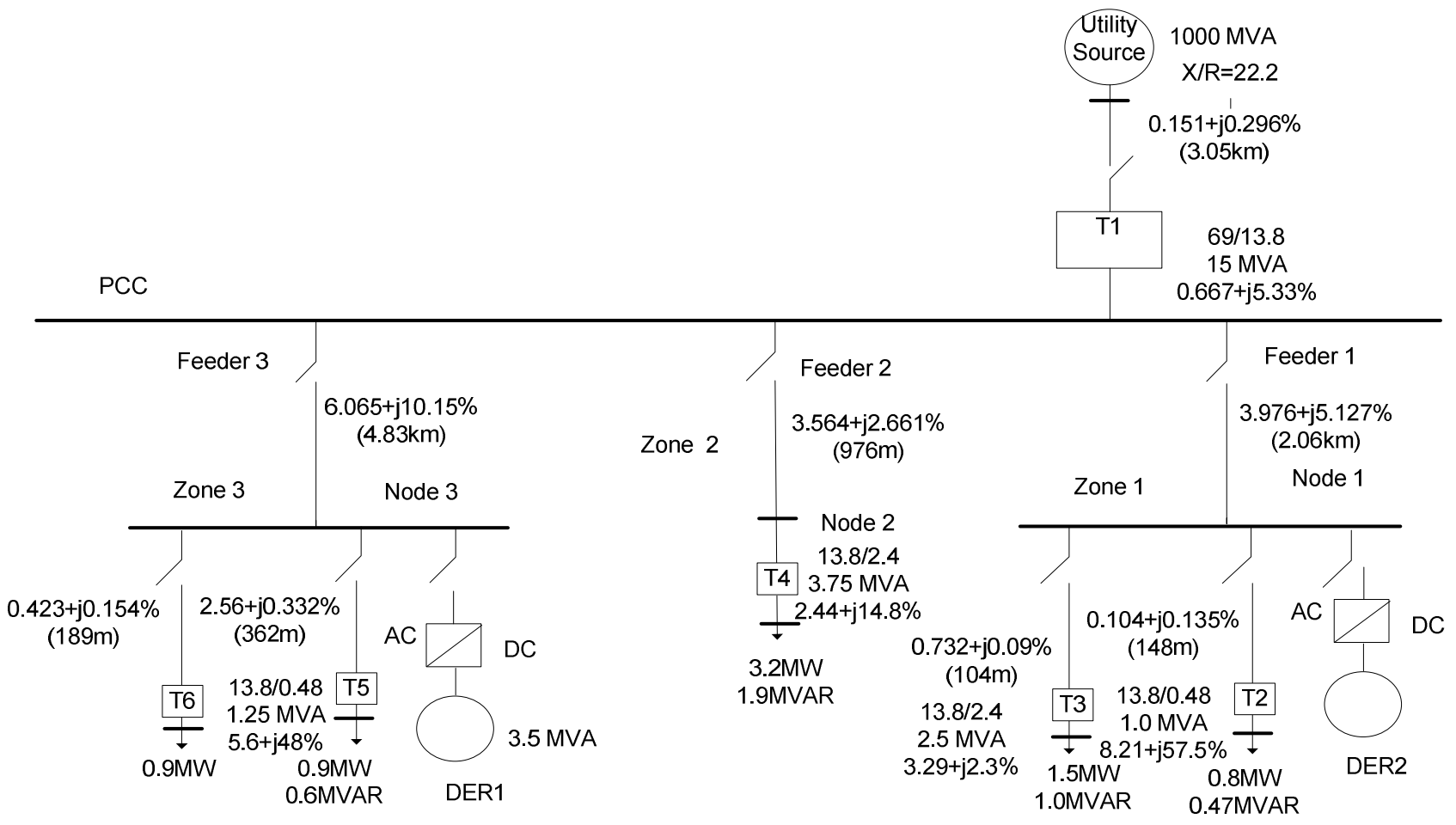

Fig. 5. Single line diagram of the test microgrid system 